\title{
Postrecurrence Clinical Outcome of Patients with Stage I Epithelial Ovarian Cancer Who Underwent Fertility-Sparing Surgery Compared to Those with Radical Surgery
}

\author{
Hiroaki Kajiyama $^{1 *}$, Kiyosumi Shibata ${ }^{1}$, Mika Mizuno ${ }^{1}$, Eiko Yamamoto ${ }^{1}$, \\ Michiyasu Kawai ${ }^{2}$, Tetsuro Nagasaka ${ }^{3}$, Fumitaka Kikkawa ${ }^{1}$ \\ ${ }^{1}$ Department of Obstetrics and Gynecology, Nagoya University Graduate School of Medicine, Nagoya, Japan \\ ${ }^{2}$ Department of Obstetrics and Gynecology, Toyohashi Municipal Hospital, Toyohashi, Japan \\ ${ }^{3}$ Department of Medical Laboratory Sciences, School of Health Science, \\ Nagoya University Graduate School of Medicine, Nagoya, Japan \\ Email: *kajiyama@med.nagoya-u.ac.jp
}

Received November 5, 2012; revised December 6, 2012; accepted December 15, 2012

\begin{abstract}
Background: To examine the difference in the survival of patients with recurrent ovarian cancer who received fertility-sparing surgery (FSS) and those receiving radical surgery. Methods: Clinicopathologic data on a total of 90 patients with stage I recurrent ovarian cancer collected under the central pathological review system were subjected to survival analyses. Patients were divided into 2 groups: 1) FSS $(\mathrm{N}=11) ; 2)$ Radical $(\mathrm{N}=79)$. Results: Five-year overall survival rates of patients in the two groups were as follows: $40.8 \%$ (FSS)/44.2\% (Radical), respectively. There was no significant difference in overall survival among the groups $(\mathrm{P}=0.887)$. Additionally, three-year postrecurrence survival rates of patients in the two groups were $24.8 \%$ (FSS) and $25.3 \%$ (Radical) $(\mathrm{P}=0.730)$. Furthermore, we accumulated 137 patients $\{\mathrm{FSS}(\mathrm{N}=58)$, Radical group $(\mathrm{N}=79)\}$ with stage I recurrent ovarian cancer from the current study and six representative reports in the literature. Patients who experienced recurrence in the remaining ovary alone (FSS) showed a more favorable prognosis than those who had extra-ovarian site recurrence (overall survival: $\mathrm{P}=0.021$, postrecurrence survival: $\mathrm{P}=0.069$ ). Conclusions: Although our retrospective analysis was very preliminary, we could propose the hypothesis that patients with stage I recurrent ovarian cancer who undergo FSS may not show poorer survival rates than patients who receive radical surgery.
\end{abstract}

Keywords: Recurrent Ovarian Cancer; Fertility-Sparing Surgery; Overall Survival; Postrecurrence Survival; Recurrence Site

\section{Introduction}

In the Cancer Statistics of 2008, it was estimated that 225,900 women were diagnosed with epithelial ovarian cancer (EOC), and 140,200 died of the disease worldwide [1]. A number of investigations have estimated that $3 \%-17 \%$ of all EOCs occur in patients at reproductive age [2-6]. In Japan, approximately $10 \%$ of women with EOC were reported to be diagnosed at childbearing age [7]. In this young generation, the preservation of reproductive and/or female endocrine functions is a crucial problem.

Thus, since most young women with early-stage EOC desire to preserve their reproductive functions, fertility-sparing surgery (FSS) has been selected in those pa-

"Corresponding author. tients for stage I invasive EOC under several criteria, including patients' strong wish, substage, histological type, and tumor grade. However, the amount of evidence has not been sufficient to answer whether, in selecting FSS, the risk of recurrence or death may be increased. In our recent study, to assess the appropriateness of FSS, we compared survival between 74 patients who had undergone FSS and 498 patients receiving radical surgery. On comparison, we revealed that there was no difference in the overall and recurrence-free survival between the two groups, either on uni- or multivariable analysis [8]. Nevertheless, here, we are again reminded of the question of how favorable the prognosis is of recurrent EOC patients receiving FSS. With regard to women with recurrent EOC who have undergone FSS, there has been limited information yielding by previous studies. 
In the present study, in an attempt to answer the above-mentioned questions, we retrospectively examined the difference in the overall and postrecurrence survival of patients with stage I recurrent EOC who had received conservative and radical surgery. Furthermore, in accumulating patients from the current study and previous literature, we also investigated whether there was an association between the recurrence sites and clinical outcome. Herein, the novel concept of FSS in stage I EOC patients is proposed.

\section{Materials and Methods}

\subsection{Patients}

A variety of malignant ovarian neoplasms have been accumulated by the Tokai Ovarian Tumor Study Group, consisting of Nagoya University and affiliated cooperating institutions, under the central pathological review system since 1986. Up to the present, more than 1500 cases of EOC have been registered and analyzed in this group. From these cases, there were 584 patients with stage I EOC, excluding borderline malignancy, that we could obtain available data on regarding management and outcomes with a sufficient length of follow-up. During observation, 90 patients experienced recurrence, including 11 and 79 patients who had received FSS and conventional radical surgery, respectively (Figure 1). Recurrence rates in the FSS and RS groups were $14.7 \%$ $(11 / 75)$ and $15.5 \%$ (79/509), respectively. In the current study, the above 90 patients with stage I recurrent EOC were retrospectively analyzed. In principle, we carried out FSS for those patients under the concrete criteria below 8: 1) had histologically confirmed stage I EOC; 2 ) were less than or equal to 40 years of age at the time of the initial diagnosis; 3 ) strongly desired to retain fertility; 4) in a preoperative counseling session, these women were informed of the possible risks and benefits of FSS, and signed a consent form. This study was approved by the ethics committee of Nagoya University. Data were collected from the medical records and at clinical follow-up visits. Twenty-one patients were excluded from this study when they showed insufficient clinical data $(\mathrm{N}$ $=16$ ) or were lost to follow-up immediately after surgery $(\mathrm{N}=5)$. The histological types and tumor grade were assigned according to the criteria of the World Health Organization (WHO). The stage was based on FIGO (International Federation of Gynecology and Obstetrics: 1988). Histological slides were reviewed by one of the authors under a central pathological review system with no knowledge of the patients' clinical data.

The surgical procedure when we selected FSS was described previously [8]. In the present study, we defined FSS for EOC as at least conservation of the uterus, contralateral ovary, and fallopian tube with full peritoneal staging (cytology of peritoneal washing or ascites, care-

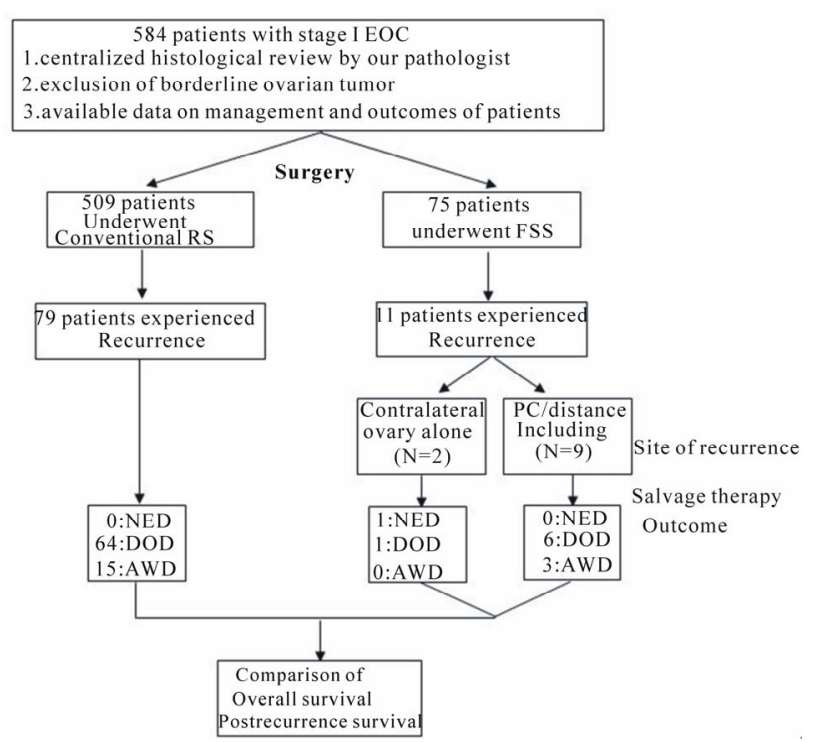

Figure 1. Flow chart of the patients included in the study. EOC: epithelial ovarian cancer, FSS: fertility-sparing surgery, RS: radical surgery, NED: no evidence of disease, DOD: died of the disease, AWD: alive without disease, PC: peritoneal cavity.

ful palpation and inspection throughout the peritoneal cavity, and, if necessary, multiple peritoneal biopsies). In patients who had undergone radical surgery as an initial treatment, the following were carried out in principle: hysterectomy and bilateral salpingo-oophorectomy with peritoneal staging (peritoneal washing, omentectomy, multiple peritoneal biopsies, and the removal of peritoneal implants) with retroperitoneal lymphadenectomy or sampling. In part of the patients at too advanced an age (e.g., over eighty years old), or with severe complications, retroperitoneal lymphadenectomy was exceptionally omitted. If retroperitoneal lymphadenectomy was omitted, the absence of a swollen lymph node more than $1 \mathrm{~cm}$ in diameter was confirmed by preoperative CT scan; however, if present, palpable nodes were appropriately sampled.

In all, 78 patients $(86.7 \%)$ were treated postoperatively with 3 to 6 cycles of adjuvant platinum-based chemotherapy (50: taxane plus platinum, 28: conventional platinum-based chemotherapy). Twelve (13.3\%) did not receive adjuvant chemotherapy due to severe comorbidities, the patients' wishes, being within the criterion of omission (stage IA/grade 1), and the decision of each institution.

\subsection{Follow-Up and Analysis}

At the end of treatment, all patients underwent a strict follow-up, consisting of clinical checkups such as a pelvic examination, transvaginal and/or transabdominal ultrasonographic scan, CA125 evaluation, and periodic plain and/or contrast-enhanced multislice CT scan throughout 
the trunk. The overall survival (OS) was defined as the time between the date of surgery and last date of followup or death due to EOC. The postrecurrence survival was defined as the time interval between the date of recurrence and last date of follow-up or death due to the disease. The distributions of clinicopathologic events were evaluated using the Chi-square or Fisher's exact tests. Univariate survival analysis was based on the KaplanMeier method. Comparison between the survival curves was conducted using the Log-rank test. A P-value of $<0.05$ was considered significant.

\section{Results}

\subsection{Clinical Outcome}

Patients' characteristics are summarized in Table 1. The median follow-up for all surviving patients was 36.4 (4.6 214.6) months. The stage distribution was as follows: patients who had received FSS (Radical): IA: 2 (12), IB: 1 (3), and IC: 8 (64). In addition, the distribution of the histological type was as follows: FSS (Radical): serous: 2 (12), clear-cell: 1 (37), endometrioid: 3 (13), mucinous: 4 (14), and others: 1 (3). Moreover, the rates of positive cytology/washing were $45.5 \%$ (5/11) in the FSS group, and $32.9(26 / 79)$ in the radical surgery group. There was no difference in the distribution of the listed clinicopathologic factors excluding age. A flow chart of patients included in the study is shown in Figure 1. The site of recurrence in patients who had undergone FSS was: 2 in the contralateral ovary, and the 9 in distant organs and/or the peritoneal cavity.

As a consequence of postrecurrence salvage therapy, including second radical or cytoreductive surgery with front-/second-line platinum-based chemotherapy, the clinical outcomes in patients who had undergone FSS (Radical) were as follows: no evidence of the disease: 1 (0), alive with the disease: 3 (15), and died of the disease: 7 (64).

Next, we compared the OS between the FSS and radical surgery groups. The 5 -year OS rates were $40.8 \%$ (FSS) and $44.2 \%$ (Radical). The patients in the FSS group did not show a poorer OS than those in the radical surgery group $(\mathrm{P}=0.887)$ (Figures 2 and 3 ) shows the postrecurrence survival curves of patients in both groups. The 3-year postrecurrence survival rates of patients in FSS and radical surgery groups were $24.8 \%$, and $25.3 \%$, respectively. The postrecurrence survival of patients in the FSS group was the same as that of patients in the radical surgery group $(\mathrm{P}=0.730)$.

To eliminate selection bias from a number of clinicopathologic factors as thoroughly as possible, the age $(\leq 40$ vs. $>40$ ), FIGO stage (IA vs. IB-C), surgical procedure (FSS vs. Radical), histological type (clear-cell vs. nonclear-cell), and postoperative adjuvant chemotherapy
Table 1. Patients' characteristics.

\begin{tabular}{|c|c|c|c|c|c|c|c|}
\hline & Total & & & $S$ & & SS & $\mathrm{P}$ \\
\hline & $\mathrm{N}$ & $\%$ & $\mathrm{~N}$ & $\%$ & $\mathrm{~N}$ & $\%$ & \\
\hline Total & 90 & & 79 & & 11 & & \\
\hline $\begin{array}{c}\text { Median } \\
\text { follow-up (range) }\end{array}$ & $\begin{array}{c}36.4 \\
(4.6-214.6)\end{array}$ & & & & & & \\
\hline Age (y) & & & & & & & $<0.0001$ \\
\hline$\leq 40$ & 21 & 23.3 & 10 & 12.7 & 11 & 100 & \\
\hline$>40$ & 69 & 76.7 & 69 & 87.3 & 0 & 0 & \\
\hline FIGO stage & & & & & & & 0.688 \\
\hline Ia & 14 & 15.6 & 12 & 15.2 & 2 & 18.2 & \\
\hline $\mathrm{Ib}$ & 4 & 4.4 & 3 & 3.8 & 1 & 9.1 & \\
\hline Ic & 72 & 80.0 & 64 & 81.0 & 8 & 72.7 & \\
\hline Histological type & & & & & & & 0.128 \\
\hline Serous & 14 & 15.6 & 12 & 15.2 & 2 & 18.2 & \\
\hline Clear-cell & 38 & 42.2 & 37 & 46.8 & 1 & 9.1 & \\
\hline Endometrioid & 16 & 17.8 & 13 & 16.5 & 3 & 27.3 & \\
\hline Mucinous & 18 & 20.0 & 14 & 17.7 & 4 & 36.4 & \\
\hline Others\#1 & 4 & 4.4 & 3 & 3.8 & 1 & 9.1 & \\
\hline Differentiation & & & & & & & 0.096 \\
\hline G1 & 27 & & 21 & 26.6 & 6 & 54.5 & \\
\hline G2 & 15 & & 13 & 16.5 & 2 & 18.2 & \\
\hline G3 & 10 & & 8 & 10.1 & 2 & 18.2 & \\
\hline $\mathrm{NA \# 2}$ & 38 & & 37 & 46.8 & 1 & 9.1 & \\
\hline $\begin{array}{c}\text { Peritoneal } \\
\text { cytology/washing }\end{array}$ & & & & & & & 0.412 \\
\hline Negative & 59 & 65.6 & 53 & 67.1 & 6 & 54.5 & \\
\hline Positive & 31 & 34.4 & 26 & 32.9 & 5 & 45.5 & \\
\hline $\begin{array}{l}\text { Platinum based } \\
\text { chemotherapy }\end{array}$ & & & & & & & 0.659 \\
\hline Absent & 12 & 13.3 & 11 & 13.9 & 1 & 9.1 & \\
\hline Present & 78 & 86.7 & 68 & 86.1 & 10 & 90.9 & \\
\hline
\end{tabular}

FIGO: international federation of gynecology and obstetrics (1988), FSS: fertility-sparing surgery, RS: conventional radical surgery, \#1: mixed epithelial adenocarcinoma and undifferentiated type, \#2: not assessed (clearcell carcinoma).

(absent vs. present) were entered into the multivariable analyses (Table 2). Among those factors, the stage was a significantly or marginal-significantly poorer prognostic factor for overall $(\mathrm{P}=0.040)$ and postrecurrence survival $(\mathrm{P}=0.051)$. However, the surgical procedure was not (OS, HR: $1.012,95 \%$ CI: $0.379-2.698, \mathrm{P}=0.982$; Postrecurrence survival, HR: $1.298,95 \%$ CI: 0.483 $3.493, \mathrm{P}=0.605)$. 


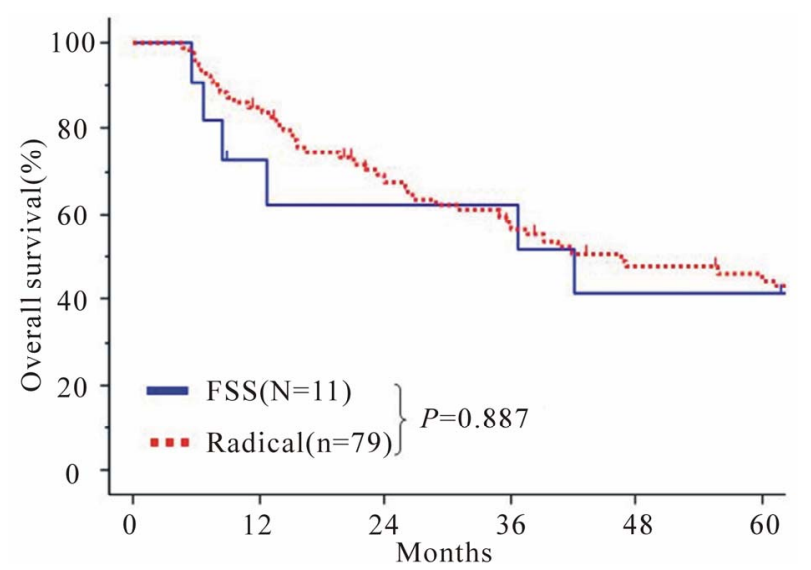

Figure 2. Kaplan-Meier estimated overall survival of patients with stage I recurrent EOC who had undergone FSS and radical surgery. Solid line: FSS $(n=11)$, dotted line: radical surgery $(n=79)$. P-values between the two groups on univariate analysis are listed under the survival curves.

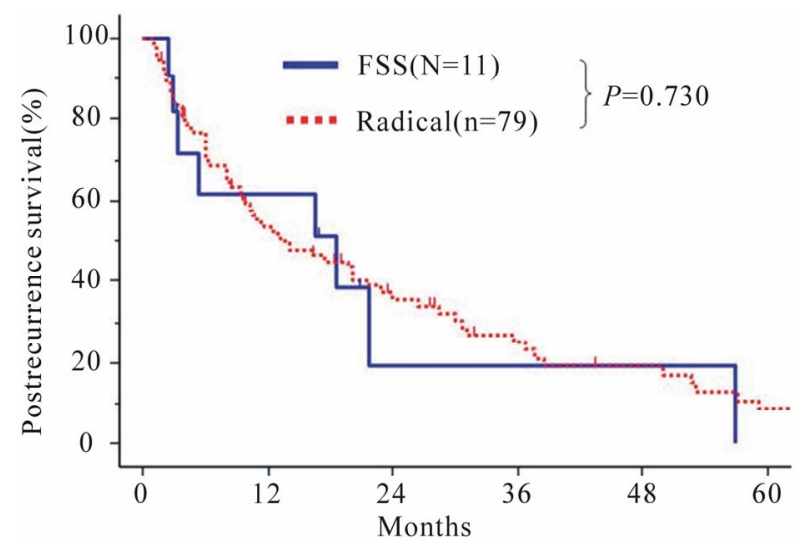

Figure 3. Kaplan-Meier estimated postrecurrence survival of patients with stage I recurrent EOC who had undergone FSS and radical surgery. Solid line: FSS $(n=11)$, dotted line: radical surgery $(n=79)$.

\subsection{Survival Analysis in Patients with Recurrent EOC, with Evidence from the Current Study and Representative Literature: FSS vs. Radical Surgery}

Previous reports regarding recurrent EOC patients treated conservatively are extremely limited. However, in six previous studies reporting patients who had undergone FSS, clinical outcomes, including survival data, were described in detail [9-14]. A total of 138 patients with stage I recurrent EOC were accumulated from both the current study and literature \{59: FSS (11: current study, 48: in the literature) and 79: Radical surgery (current study) $\}$. The characteristics and distribution of clinicopathologic factors and oncologic outcomes of those patients are presented in Table 3. Among 58 in 59 patientswho underwent FSS and with clinical information on recurrence sites, there were 16 patients $(27.6 \%)$ who
Table 2. Multivariable analyses of clinicopathologic parameters in relation to survival of patients with stage I recurrent EOC.

\begin{tabular}{|c|c|c|c|c|}
\hline & OS: HR $(95 \% \mathrm{CI})$ & $\mathrm{P}$ & PRS: HR $(95 \% \mathrm{CI})$ & $\mathrm{P}$ \\
\hline \multicolumn{5}{|l|}{ Age } \\
\hline$\leq 40$ & 1 & & 1 & \\
\hline$>40$ & $\begin{array}{c}1.123 \\
(0.516-2.445)\end{array}$ & 0.770 & $\begin{array}{c}0.1050 \\
(0.483-2.282)\end{array}$ & 0.902 \\
\hline \multicolumn{5}{|l|}{ FIGO stage } \\
\hline IA & 1 & & 1 & \\
\hline $\mathrm{IB} / \mathrm{C}$ & $\begin{array}{c}2.324 \\
(1.037-5.208)\end{array}$ & 0.040 & $\begin{array}{c}2.376 \\
(0.997-5.664)\end{array}$ & 0.051 \\
\hline \multicolumn{5}{|l|}{ Surgery } \\
\hline Radical & 1 & & 1 & \\
\hline FSS & $\begin{array}{c}1.012 \\
(0.379-2.698)\end{array}$ & 0.982 & $\begin{array}{c}1.298 \\
(0.483-3.493)\end{array}$ & 0.605 \\
\hline \multicolumn{5}{|l|}{ Histology } \\
\hline Clear-cell & 1 & & 1 & \\
\hline Non clear-cell & $\begin{array}{c}0.790 \\
(0.445-1.403)\end{array}$ & 0.790 & $\begin{array}{c}0.725 \\
(0.410-1.281)\end{array}$ & 0.268 \\
\hline \multicolumn{5}{|l|}{$\begin{array}{l}\text { Platinum-based } \\
\text { chemotherapy }\end{array}$} \\
\hline Present & 1 & & 1 & \\
\hline Unknown & $\begin{array}{c}2.867 \\
(0.708-11.619)\end{array}$ & 0.140 & $\begin{array}{c}2.377 \\
(0.565-10.001)\end{array}$ & 0.238 \\
\hline Absent & $\begin{array}{c}1.105 \\
(0.478-2.555)\end{array}$ & 0.816 & $\begin{array}{c}1.281 \\
(0.495-3.315)\end{array}$ & 0.609 \\
\hline
\end{tabular}

EOC: epithelial ovarian cancer, OS: overall survival, PRS: postrecurrence survival, HR: hazard ratio, CI: confidential interval, FIGO: International Federation of Gynecology and Obstetrics, FSS: fertility-sparing surgery.

experienced recurrence in the contralateral ovary alone. In contrast, in the remaining 42 patients $(72.4 \%)$, the site of recurrence was throughout the peritoneal cavity (PC) and/or distant organs. We further analyzed them by stratifying patients by recurrence site, as follows: 1) FSS (ovary); 2) FSS (PC/distant); and 3) Radical surgery. Figure 4(a) shows OS curves of the three groups. Patients in the FSS (ovary) group showed a more favorable OS than those in the FSS (PC/distant) $(\mathrm{P}=0.021)$ or radical surgery group $(\mathrm{P}<0.0001)$. Furthermore, regarding postrecurrence survival, a similar tendency was noted among the three groups FSS (ovary) vs. FSS (PC/distant), $\mathrm{P}=0.069$ : FSS (ovary) vs. Radical, $\mathrm{P}<0.0001$ \} (Figure 4(b)).

\section{Discussion}

Numerous authors have reported the clinical course of patients who have undergone FSS, and so this surgical procedure may be appropriate for stage I EOC under individual criteria, including the substage, histological 
Table 3. Clinical outcome in patients with stage I recurrent EOC, with evidence from the current study and representative literature.

\begin{tabular}{|c|c|c|c|c|c|c|c|c|c|c|c|}
\hline Report & Year & Total & $\operatorname{Rec}$ & Histology & Stage & & Site of Rec. & & Survival $^{\# 1}$ & PRS & Status \\
\hline & & No. & No. & & & RO & PC/distant & NR & (M) & (M) & \\
\hline Zanetta & 1997 & 56 & 5 & S: (2), M: (1), E: (2) & IA: (4), IC: (1) & 1 & 4 & 0 & $8-44$ & $N A$ & $\begin{array}{c}\text { NED: (1), AWD: (1), } \\
\text { DOD: (3) }\end{array}$ \\
\hline Schilder & 2002 & 52 & 5 & $\begin{array}{c}\text { S: (1), M:(2), E: (1), } \\
\text { Mi: (1) }\end{array}$ & IA: (4), IC: (1) & 3 & 2 & 0 & $13-97$ & $4-40$ & NED: (3), DOD: (2) \\
\hline Marpeau & 2008 & 33 & 9 & $\begin{array}{l}\text { S: (2), M: (4), E: (1), } \\
\text { C: (1), Mi: (1) }\end{array}$ & IA: (6), IC: (3) & 4 & 5 & 0 & $9-272$ & $3-260$ & $\begin{array}{l}\text { NED: (3), AWD: (2), } \\
\text { DOD: (3), Un: (1) }\end{array}$ \\
\hline Park & 2008 & 59 & 9 & $\begin{array}{c}\text { M: (5), E: (1), C: (2), } \\
\text { Mixed: (1) }\end{array}$ & IA: (5), IC: (4) & 1 & 8 & 0 & $11-133$ & $5-79$ & $\begin{array}{c}\text { NED: (2), AWD: (3), } \\
\text { DOD: (4) }\end{array}$ \\
\hline Anchezar & 2009 & 14 & 2 & M: (1), E: (1) & IA: (2) & 0 & 2 & 0 & 16,78 & 9,15 & NED: (1), DOD: (1) \\
\hline Satoh & 2010 & 211 & 18 & $\begin{array}{c}\text { S: (3), M: (6), E: (4), } \\
\text { C: (5) }\end{array}$ & IA: (7), IC: (11) & 5 & 12 & 1 & $6-256$ & $5-231$ & $\begin{array}{l}\text { NED: (8), AWD: (5), } \\
\text { DOD: (5) }\end{array}$ \\
\hline $\begin{array}{c}\text { Current } \\
\text { report }\end{array}$ & $(-)$ & 75 & 11 & $\begin{array}{c}\text { S: (2), M: (4), E: (3), C: } \\
\text { (1), Un (1) }\end{array}$ & $\begin{array}{l}\text { IA: (2), IB: (1), } \\
\text { IC: (8) }\end{array}$ & 2 & 9 & 0 & $6-196$ & $3-57$ & $\begin{array}{l}\text { NED: (1), AWD: (3), } \\
\text { DOD: (7) }\end{array}$ \\
\hline Total & & 500 & 59 & & & 16 & 42 & 1 & & & \\
\hline
\end{tabular}

Rec: recurrence, S: serous, M: mucinous, E: endometrioid, C: clear-cell, Mi: mixed, Un: undifferentiated, PRS: postrecurrence survival, RO: remaining ovary, PC: peritoneal cavity, NED: no evidence of disease, AWD: alive with disease, DOD: died of disease.

type, and tumor grade [10-13,15,16]. Nevertheless, owing to the uncertain risk of leaving an occult tumor in the remaining contralateral ovary or threat of recurrence anywhere, we frequently question choosing FSS for all patients with stage I EOC. In this regard, two principal questions underlie: when we select the surgical procedure, is the postrecurrence survival altered if we select FSS? Or, is their prognosis influenced if the site of recurrence differs? To answer these questions as fully as possible, we have reported an investigation comparing the prognosis of patients receiving FSS with those who underwent radical surgery from various points of view, including tumor differentiation, histology, and the substage of stage I $[8,17,18]$. As a result, we have shown that there was no difference in the overall and recurrence-free survival of patients between the two groups, although there were several limitations reflecting the retrospective nature 8 . However, there arose a new question of whether recurrence cases who had undergone FSS showed a poorer survival than those who had received radical surgery and experienced recurrence. In the current study, we also showed that there was no significant difference in overall and postrecurrence survival between the two groups. Since it was a very small-scale examination, we could not derive a definite conclusion from this. However, to our knowledge, there has been no study comparing the survival time between them. At least, we could propose the hypothesis that the prognosis of patients with recurrent EOC undergoing FSS may be the same as that of those receiving radical surgery. To verify the appropriateness of FSS for early-stage EOC, we hope that it is confirmed by further examination.
Based on a number of studies reporting FSS in patients with recurrent EOC, the recurrence site was divided into two major parts: 1) remaining contralateral ovary alone, and extra-ovarian organ including sites, such as the peritoneal cavity and/or distant organs. According to the large-scale retrospective report by Satoh et al., five patients with EOC who received FSS experienced recurrence within the remaining ovary. Then, they were treated with salvage surgery and showed no evidence of disease [12]. In addition, a number of such patients in the literature were consequently rescued similarly. Thus, we expected that these patients display better postrecurrence survival following salvage treatment compared with those with extra-ovarian recurrence sites. To confirm this hypothesis, we further analyzed a total of 137 patients with stage I recurrent EOC who had undergone FSS (N = 58 ) or radical surgery $(\mathrm{N}=79)$, accumulating them from the current study and representative literature [9-14]. As a consequence, in patients of the FSS group, a markedly favorable prognosis was noted, as shown in the survival curve, when recurrence was observed in the remaining ovary alone. In addition, prognostic inferiority was not observed in the patients who experienced recurrence in extra-ovarian sites after FSS to those who underwent radical surgery. Why did patients who developed recurrence show comparatively favorable survival rates? If the recurrence originated from the occult metastasis via peritoneal dissemination and/or lymphovascular flow, it would be a poorer oncologic outcome than we observed in the current study. Although it is a personal hypothesis, the remaining ovary-confined relapse may be due to the de novo tumor generation. We would like to evaluate this 


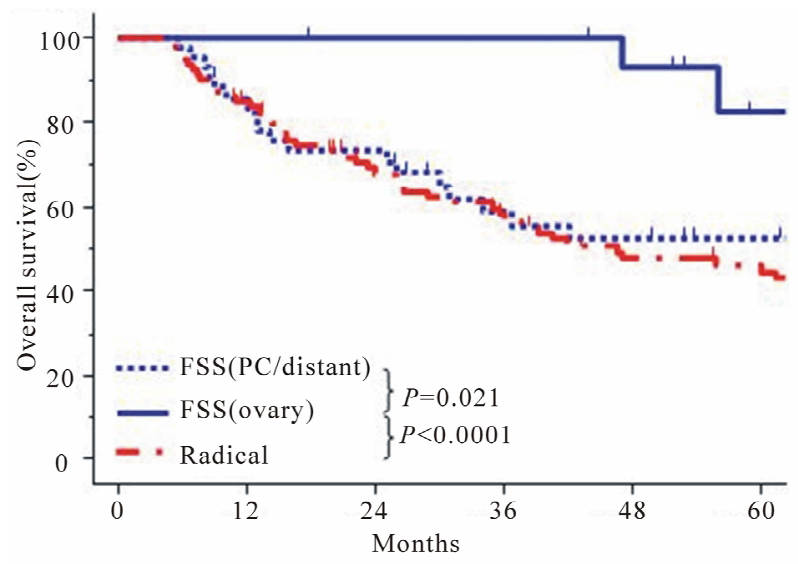

(a)

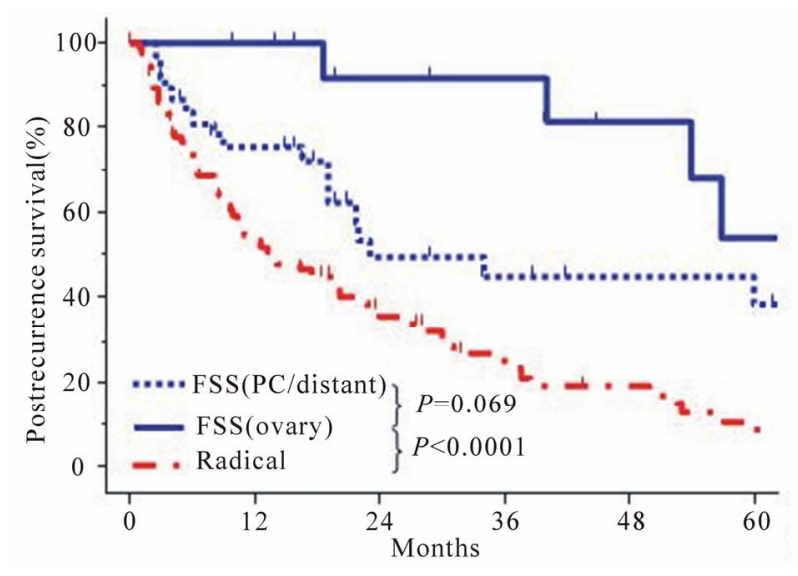

(b)

Figure 4. (a) Kaplan-Meier estimated overall survival of 137 recurrent EOC patients with definite information on recurrence site, accumulated from the current study and six representative reports from the literature [9-14]. Confining to this analyses, OS was defined as the interval between the last treatment (surgery or last treatment) and last follow-up or death. Each group stratified by the initial surgical procedure and recurrence site is as follows: FSS (ovary alone): ( $N=16$, solid line), FSS (PC/distant): ( $N=42$, dotted line), and Radical ( $\mathrm{N}=79$, dashed line); (b) Kaplan-Meier estimated postrecurrence survival of 132 patients with stage I recurrent EOC patients accumulated from the current study and five representative literature [9-12,14]. Each group stratified by the initial surgical procedure and recurrence site is as follows: FSS (ovary alone): ( $\mathrm{N}=15$, solid line), FSS (PC/distant): ( $N=38$, dotted line), and Radical $(N=79$, dashed line).

in a future study.

In summary, a novel finding regarding FSS for earlystage EOC is proposed. At least, we presented a hypothesis that patients who received FSS may not necessarily exhibit poorer postrecurrence survival than those who underwent radical surgery. Our retrospective analysis was too preliminary and had a lot of limitations, such as a small number of cases, lack of sufficient power, and variable follow-up length, to reach a definite conclusion.
Furthermore, in evaluating FSS for Stage I EOC, several clinicopathologic indicators, including substage, tumor grade, and histological type, overlap with one another and present an unexpectedly complicated picture. It is possible that patients who selected FSS potentially had a favorable clinicopathologic factors than those with radical surgery. Essentially, the randomized controlled trial is a solution to this problem; however, it is actually very difficult to perform because of ethical aspect. Nevertheless, indeed, women who face a serious problem are at a loss regarding how long patients underwent FSS survives once they experience recurrence and what they should choose. Our current data could be hypothesis-generating for a gynecologic oncologist and patient who are confronted with this crucial issue. To assess the further appropriateness of FSS, we would like to accumulate more experiences of this tumor and reconfirm the current results in the future.

\section{Acknowledgements}

We sincerely thank Drs. Y. Kinoshita (Ogaki Municipal Hospital), K. Sakakibara (Okazaki Municipal Hospital), A. Takeda (Gifu Prefectural Tajimi Hospital), O. Yamamuro (Japanese Red Cross Nagoya Second Hospital), K. Mizuno (Japanese Red Cross Nagoya first Hospital), T. Nakanishi (Aichi Cancer Center Hospital), and K. Matsuzawa (Anjyo Kosei Hospital) who collaborated in data collection.

\section{REFERENCES}

[1] A. Jemal, F. Bray, M. M. Center, J. Ferlay, E. Ward and D. Forman, "Global Cancer Statistics," A Cancer Journal for Clinicians, Vol. 61, No. 2, 2011, pp. 69-90. doi:10.3322/caac. 20107

[2] L. R. Duska, Y. C. Chang, C. E. Flynn, A. H. Chen, A. Goodman, A. F. Fuller and N. Nikrui, "Epithelial Ovarian Carcinoma in the Reproductive Age Group," Cancer, Vol. 85 , No. 12 , 1999, pp. 2623-2629.

doi:10.1002/(SICI)1097-0142(19990615)85:12<2623::AI D-CNCR19>3.0.CO;2-O

[3] S. C. Plaxe, P. S. Braly, J. L. Freddo, E. McClay, S. Kirmani and S. B. Howell, "Profiles of Women Age 30 - 39 and Age Less than 30 with Epithelial Ovarian Cancer," Obstetrics and Gynecology, Vol. 81, No. 5, 1993, pp. 651-654.

[4] M. Rodriguez, H. N. Nguyen, H. E. Averette, A. J. Steren, M. A. Penalver, T. Harrison and B. U. Sevin, "National Survey of Ovarian Carcinoma XII. Epithelial Ovarian Malignancies in Women Less than or Equal to 25 Years of Age," Cancer, Vol. 73, No. 4, 1994, pp. 1245-1250. doi:10.1002/1097-0142(19940215)73:4<1245::AID-CNC $\underline{\text { R2820730419>3.0.CO;2-5 }}$

[5] K. D. Swenerton, T. G. Hislop, J. Spinelli, J. C. LeRiche, N. Yang and D. A. Boyes, "Ovarian Carcinoma: A Multivariate Analysis of Prognostic Factors," Obstetrics and 
Gynecology, Vol. 65, No. 2, 1985, pp. 264-270.

[6] H. Smedley and K. Sikora, "Age as a Prognostic Factor in Epithelial Ovarian Carcinoma," BJOG: An International Journal of Obstetrics \& Gynaecology, Vol. 92, No. 8, 1985 , pp. 839-842. doi:10.1111/j.1471-0528.1985.tb03056.x

[7] Acta Obstetrica et Gynaecologica Japonica, Vol. 63, 2011, pp. 1076-1096.

[8] H. Kajiyama, K. Shibata, M. Mizuno, T. Umezu, S. Suzuki, A. Nawa, M. Kawai, T. Nagasaka and F. Kikkawa, "Long-Term Survival of Young Women Receiving Fertility-Sparing Surgery for Ovarian Cancer in Comparison with Those Undergoing Radical Surgery," British Journal of Cancer, Vol. 105, 2011, pp. 1288-1294. doi: $10.1038 /$ bjc. 2011.394

[9] O. Marpeau, J. Schilder, Y. Zafrani, C. Uzan, S. Gouy, C. Lhomme and P. Morice, "Prognosis of Patients Who Relapse after Fertility-Sparing Surgery in Epithelial Ovarian Cancer," Annals of Surgical Oncology, Vol. 15, No. 2, 2008, pp. 478-483. doi:10.1245/s10434-007-9651-x

[10] J. M. Schilder, A. M. Thompson, P. D. DePriest, F. R. Ueland, M. L. Cibull, R. J. Kryscio, S. C. Modesitt, K. H. Lu, J. P. Geisler, R. V. Higgins, P. M. Magtibay, D. E. Cohn, M. A. Powell, C. Chu, F. B. Stehman and J. van Nagell, "Outcome of Reproductive Age Women with Stage IA or IC Invasive Epithelial Ovarian Cancer Treated with Fertility-Sparing Therapy," Gynecologic and Oncology, Vol. 87, No. 1, 2002, pp. 1-7. doi:10.1006/gyno.2002.6805

[11] J. Y. Park, D. Y. Kim, D. S. Suh, J. H. Kim, Y. M. Kim, Y. T. Kim and J. H. Nam, "Outcomes of Fertility-Sparing Surgery for Invasive Epithelial Ovarian Cancer: Oncologic Safety and Reproductive Outcomes," Gynecologic Oncology, Vol. 110, No. 3, 2008, pp. 345-353. doi:10.1016/i.ygyno.2008.04.040

[12] T. Satoh, M. Hatae, Y. Watanabe, N. Yaegashi, O. Ishiko, S. Kodama, S. Yamaguchi, K. Ochiai, M. Takano, H. Yokota, Y. Kawakami, S. Nishimura, D. Ogishima, S. Nakagawa, H. Kobayashi, T. Shiozawa, T. Nakanishi, T. Kamura, I. Konishi and H. Yoshikawa, "Outcomes of
Fertility-Sparing Surgery for Stage I Epithelial Ovarian Cancer: A Proposal for Patient Selection," Journal of Clinical Oncology, Vol. 28, No. 10, 2010, pp. 1727-1732. doi:10.1200/JCO.2009.24.8617

[13] G. Zanetta, S. Chiari, S. Rota, G. Bratina, A. Maneo, V. Torri and C. Mangioni, "Conservative Surgery for Stage I Ovarian Carcinoma in Women of Childbearing Age," British Journal of Obstetrics and Gynaecology, Vol. 104, No. 9, 1997, pp. 1030-1035. doi:10.1111/j.1471-0528.1997.tb12062.x

[14] J. P. Anchezar, J. Sardi and A. Soderini, "Long-Term Follow-Up Results of Fertility Sparing Surgery in Patients with Epithelial Ovarian Cancer," Journal of Surgical Oncology, Vol. 100, No. 1, 2009, pp. 55-58. doi: $10.1002 /$ jso. 21297

[15] P. Morice, F. Wicart-Poque, A. Rey, J. El-Hassan, P. Pautier, C. Lhomme, R. de Crevosier, C. Haie-Meder, P. Duvillard and D. Castaigne, "Results of Conservative Treatment in Epithelial Ovarian Carcinoma," Cancer, Vol. 92, No. 9, 2001, pp. 2412-2418.

doi:10.1002/1097-0142(20011101)92:9<2412::AID-CNC R1590>3.0.CO;2-7

[16] H. Kajiyama, K. Shibata, S. Suzuki, K. Ino, E. Yamamoto, K. Mizuno, K. Sakakibara, K. Matsuzawa, A. Takeda, Y. Kinoshita, M. Kawai, T. Nagasaka, A. Nawa and F. Kikkawa, "Is There Any Possibility of Fertility-Sparing Surgery in Patients with Clear-Cell Carcinoma of the Ovary?" Gynecologic Oncology, Vol. 111, No. 3, 2008, pp. 523-526. doi:10.1016/j.ygyno.2008.04.001

[17] H. Kajiyama, K. Shibata, M. Mizuno, S. Hosono, M. Kawai, T. Nagasaka and F. Kikkawa, "Fertility-Sparing Surgery in Patients with Clear-Cell Carcinoma of the Ovary: Is It Possible?" Human Reproduction, Vol. 26, No. 12, 2011, pp. 3297-3302.

[18] H. Kajiyama, K. Shibata, M. Mizuno, A. Nawa, K. Mizuno, K. Matsuzawa, M. Kawai, S. Hosono, T. Nagasaka and F. Kikkawa, "Fertility-Sparing Surgery in Young Women with Mucinous Adenocarcinoma of the Ovary," Gynecologic Oncology, Vol. 122, No. 2, 2011, pp. 334338. doi:10.1016/j.ygyno.2011.04.016 